\title{
Konjugatimpfstoff auch bei älteren Patienten wirksam
}

\author{
Eine der Hauptursachen für die ambulant erworbene Pneumonie \\ (Community Acquired Pneumonia, CAP) bei älteren Patienten ist \\ eine Infektion mit Streptococcus pneumoniae. Bei Kindern \\ verhindern Polysaccharid-Konjugatimpfstoffe bereits effektiv \\ Pneumokokken-Erkrankungen. Nun haben M. J. M. Bonten et al. \\ untersucht, ob diese Impfstoffe auch bei Erwachsenen im Alter \\ von 65 Jahren oder älter wirksam sind. \\ N Engl J Med 2015; 372: 1114-1125
}

Die CAP verursacht unter Älteren beträchtliche Morbidität und Mortalität. Am häufigsten tritt sie als nicht bakteriämische Erkrankung auf. In etwa $25 \%$ der Fälle liegt eine invasive PneumokokkenErkrankung vor. Die Wirksamkeit von Polysaccharid-Konjugatimpstoffen zur Prävention der invasiven und nicht invasiven ambulant erworbenen VakzinetypPneumokokken-Pneumonie wurde bei älteren Patienten bisher noch nicht bestimmt. Daher führten die niederländischen Forscher eine randomisierte, doppelblinde und placebokontrollierte Studie mit 84496 Erwachsenen im Alter ab 65 Jahren durch.

Sie evaluierten hierbei die Wirksamkeit eines 13-valenten Polysaccharid-Konjugatimpfstoffs (PCV13) bei der Prävention erster Episoden der invasiven und nicht invasiven ambulant erworbenen Vakzinetyp-Pneumokokken-Pneumonie sowie der nicht bakteriämischen und nicht invasiven ambulant erworbenen Pneumokokken-Pneumonie und der invasiven Pneumokokken-Erkrankung. Die Teilnehmer erhielten im Verhältnis 1:1 entweder PCV13 oder Placebo. Zur Identifizierung der Erkrankungen setzten sie Standardlabormethoden und serotypspezifische Tests zur Antigendetektion im Urin ein.

\section{Anhaltende Wirksamkeit der Vakzine \\ $\nabla$}

Wie die Analyse (per Protokoll) der ersten Infektions-Episoden, verursacht durch Vakzinetyp-Stämme, erbrachte, trat bei
49 Patienten in der PCV13- und bei 90 in der Placebo-Gruppe eine CAP auf. Das entsprach einer Vakzinewirksamkeit von 45,6\%. Eine nicht bakteriämische und nicht invasive CAP fanden die Untersucher bei 33 Patienten in der PCV13- und bei 60 in der Placebo-Gruppe (Vakzinewirksamkeit: $45 \%)$. Eine invasive PneumokokkenErkrankung trat bei 7 Patienten in der PCV13- und bei 28 Patienten in der Placebogruppe auf(Vakzinewirksamkeit: 75\%). Die Wirksamkeit hielt während der gesamten Studie mit einer mittleren Nachbeobachtungszeit von 3,97 Jahren an.

\section{Wirksamkeitsnachweis des Vakzines \\ $\nabla$}

In einer modifizierten Intent-to-treatAnalyse werteten die Untersucher die Wirksamkeit bei Teilnehmern aus, bei denen spätestens 14 Tage nach der Impfung Episoden von CAP oder invasiver Pneumokokken-Erkrankung einsetzten. Diese Analyse ergab eine ähnliche Wirksamkeit wie die Per-Protokoll-Analyse (Vakzinewirksamkeit entsprechend 37,7, 41,1 und 75,8\%). Dabei trat bei $747 \mathrm{~Pa}-$ tienten in der PCV13- und bei 787 in der Placebogruppe eine CAP jeder möglichen Ursache auf (nicht signifikante Vakzinewirksamkeit: 5,1\%). Die Anzahl schwerer Nebenwirkungen bei allen Teilnehmern betrug innerhalb eines Monats nach der Impfung 0,8\% in der PCV13-Gruppe und $0,7 \%$ unter Placebo. In beiden Gruppen lag die Anzahl an Todesfällen bei 7,1\%. Lokale Reaktionen waren in der PCV13Gruppe häufiger.

\section{Fazit}

Die fast 46\%ige Reduktion bei der Vakzinetyp-CAP nach PCV13-Immunisierung weist nach Ansicht der Autoren darauf hin, dass diese Impfung auch bei älteren Erwachsenen zu einer Reduktion der Pneumokokken-Pneumonie beitragen kann. PCV13 verhinderte zwar die Pneumokokken-, Bakteriämie- und NichtBakteriämie-CAP und invasive Pneumokokken-Erkrankungen, nicht jedoch jede CAP.

Dr. Volker Kriegeskorte, Buchloe

Ausschreibung

\section{Wissenschaftspreis in der Pneumologie}

Zweck der CAPNETZ STIFTUNG ist die Förderung der Wissenschaft und der Bildung zur Förderung wissenschaftlicher Arbeiten zum Thema „Ambulant erworbene Pneumonie“ (Community Acquired Pneumonia, CAP) und zu anderen Infektionen des unteren Respirationstraktes. Dazu gehören Studien aus den Bereichen Grundlagenforschung, mikrobiologische Forschung und jede Form klinischer Studien.Die CAPNETZ STIFTUNG vergibt alle 2 Jahre für eine herausragende Forschungsarbeit den CAPNETZ AWARD. Die einzureichenden Arbeiten kommen aus der Grundlagen- oder klinischen Forschung. Der Preis ist mit $5000 €$ dotiert und kann an einen Nachwuchswissenschaftler unter 35 Jahren für Projektmittel zukünftiger Forschungsvorhaben im Bereich respiratorischer Infektionen vergeben werden. Die Bewerbungsfrist endet am 11. Dezember 2015. Anträge können als PDF-Datei mit einem formlosen Anschreiben an die CAPNETZ STIFTUNG gerichtet werden. Die Arbeit darf nicht länger als 2 Jahre zurückliegen. Sie sollte bereits publiziert sein. Die Verleihung des Preises erfolgt im Rahmen des Hauptprogramms des 57. DGP-Kongresses im kommenden Jahr. Weitere Informationen gibt die Geschäftsführerin der CAPNETZ STIFTUNG, Grit Barten, Carl-Neuberg-Str. 1 in 30625 Hannover, Tel.: 0511/532 4434, EMail: grit.barten@capnetz.de

\section{Nach einer Mitteilung der CAPNETZ STIFTUNG, Hannover}

